\title{
Analysis and Synthesis for Linear Set-Valued Dynamically Varying Systems ${ }^{1}$
}

\author{
Stephan Bohacek and Edmond Jonckheere \\ Departments of Mathematics and Electrical Éngineering-Systems \\ University of Southern California \\ Los Angeles, CA 90089-2563 \\ bohacek@usc.edu, jonckhee@eudoxus.usc.edu
}

\begin{abstract}
Analysis and synthesis for linear systems with parameters that vary according to a set-valued map are investigated. It is shown that the optimal controllers are continuous and homogeneous, but not additive. A computational method based on approximating the nonquadratic cost by a piece-wise quadratic function is developed. Such an approximation determines stability nonconservatively and preserves the homogeneity of the controller. Computational methods for both analysis and synthesis are provided.
\end{abstract}

\section{Introduction}

Recently, controlling linear systems with varying parameters has been an active area of research. Much of this work has focused on sub-optimal linear controllers $[1],[2],[4],[11]$. However, [13] looked at nonlinear controllers for the case where the parameters can only take a finite number of values. This paper will examine the case where the system parameters can take values in an infinite set.

The general form of an LPV system is

$$
\begin{aligned}
x(k+1) & =A_{\phi(k)} x(k)+B_{\phi(k)} u(k) \\
z(k) & =\left[\begin{array}{c}
C_{\phi(k)} x(k) \\
D_{\phi(k)} u(k)
\end{array}\right] \\
\phi(k+1) & =f(\phi(k))
\end{aligned}
$$

Here, the parameters vary according to some dynamical system, $f: \Phi \rightarrow \Phi$. In the typical LPV case, all that is known about the parameter dynamics is that $f(\phi) \in \Phi$ for all $\phi \in \Phi[2]$, [4]. When $\Phi$ is a convex polytope, this LPV model has the advantage that there are very computationally efficient methods for synthesizing controllers [9]. In general, when the designer knows more about how the parameters vary than simply that they are contained in some set, these approaches will tend to be conservative. In addition, these approaches also assume that if the stage cost is quadratic, then the infinite horizon cost is quadratic and the control is linear. In general, these assumptions are not true.

\footnotetext{
${ }^{1}$ This research was supported by NSF Grant ECS-98-02594.
}

A slight specialization of the above LPV systems is accomplished by putting bounds on the rate at which the system parameters vary, i.e. $|f(\phi)-\phi|<\Delta[1],[14]$. This approach may be less conservative than the typical LPV model. However, this approach is computationally more difficult. Computational methods for analysis and synthesis for this model constitute an active area of research. This approach has shortcomings when the parameters vary drastically, for example, in the case where the controller needs to account for failures which lead to sudden changes in the system parameters. Furthermore, like the typical LPV approach, this refined LPV approach assumes that the cost is quadratic in the state and continuous in the parameters, and the control is linear. However, in [7] it was shown that the optimal $H^{\infty}$ controller may be discontinuous.

Jump linear systems [10], [12] are another type of LPV systems. Here incomplete knowledge of the dynamical system $f$ is modeled probabilistically as a Markov chain. For this approach there are also good computational methods [8]. Furthermore, for the given probabilistic model, optimal controllers are linear. A major drawback of the jump linear system approach is that only stochastic stability can be guaranteed. This form of stability does not apply to every realization of the Markov chain nor to the original nonlinear process. For example, consider a control problem where a nonlinear plant is modeled by a linear approximation around some operating point. The jump linear model makes the assumption that the operating point varies according to a Markov chain. In this case, it cannot be directly shown that the jump linear controller stabilizes every trajectory of the nonlinear plant.

Linear dynamically varying (LDV) systems are the class of LPV systems where the dynamical system $f$ is completely known [5], [7]. With such complete knowledge of the parameters dynamics, the optimal LQ and a suboptimal $H^{\infty}$ controllers can be found and are known to be linear and continuous. Furthermore, in some situations, the jump linear model described above is actually an approximation of the LDV system. In this case, since the LDV controllers are robust, it can be 
shown that the jump linear controllers are also robust as well [6]. If the parameter dynamics has certain properties, such as transitive orbits, a dense set of recurrent points, etc., then there are efficient methods for computing the optimal LDV controller. While the exact parameter dynamics may be known in some guidance and tracking problem, it is common that the exact parameter dynamics is not precisely known.

The ideal LPV approach is one which utilizes all of the a priori knowledge of the parameter dynamics. Such a system would encompass both the typical LPV approach at one extreme (almost no knowledge of the parameter dynamics) and the LDV systems at the other extreme (complete knowledge of the parameter dynamics). Such systems are linear set-valued dynamically varying (LSVDV) systems; a linear system with parameters dynamics modeled by a set-valued function, i.e. $f(\phi) \subset \Phi$. This paper will formally introduce LSVDV systems in Section 2. An explanation as to why the controller is nonlinear will be given in Section 3. The analysis and synthesis problems are discussed in Sections 4 and 5 respectively.

\section{Linear Set-Valued Dynamically Varying Systems}

A linear set-valued dynamically varying (LSVDV) system is of the form (1) where $A: \Phi \rightarrow \mathbb{R}^{n \times n}, B: \Phi \rightarrow$ $\mathbb{R}^{n \times m}, C: \Phi \rightarrow \mathbb{R}^{p \times n}$ and $D: \Phi \rightarrow \mathbb{R}^{q \times m}$ are continuous functions, and the map $f$ is a continuous set-valued map. Continuity of set-valued maps means that given $\varepsilon>0$, there exists a $\delta>0$ such that if $|\phi-\rho|<\delta$, then $d(f(\phi), f(\rho))<\varepsilon$, where

$$
d(A, B)=\max \left(\sup _{b \in B} \inf _{a \in A}(|a-b|), \sup _{a \in A} \inf _{b \in B}(|a-b|)\right)
$$

A detailed discussion of set-valued maps can be found in [3]. It is also assumed that the parameter space $\Phi$ is compact; hence, all of the above maps are uniformly continuous.

The typical LPV case is where $f(\phi)=\Phi$ for every $\phi \in \Phi$. The case where the parameters dynamics is typically known, but there may be failures which lead to abrupt changes, is given by

$$
f(\phi)= \begin{cases}\theta(\phi)_{o} & \text { no failure } \\ \theta(\phi)_{1} & \text { type } 1 \text { failure } \\ \vdots & \\ \theta(\phi)_{r} & \text { type } \mathrm{r} \text { failure }\end{cases}
$$

One situation of slowly varying parameters is given by $f(\phi)=B(\phi, \delta(\phi))$, where $B(\phi, \delta(\phi))$ is a ball around $\phi$ with radius $\delta(\phi)$. Note that the size of the ball is allowed to vary with the parameter $\phi$; hence, the rate at which the parameters vary depends of $\phi$. Another useful parameter dynamics is given by

$$
f(\phi)=\bigcup_{i=1}^{r} B\left(\theta(\phi)_{i}, \delta(\phi)_{i}\right) .
$$

Note that $f$ can be defined to account for all of the a priori knowledge of the parameter dynamics

An LSVDV system is exponentially stable if for each $\phi_{o} \in \Phi$, there exist $\alpha_{\phi_{o}}<1$ and $\beta_{\phi_{o}}<\infty$, such that $|x(k+j)|<\beta_{\phi_{o}} \alpha_{\phi_{o}}^{j}|x(k)|$. Note that this type of stability is uniform in time, but not uniform in $\phi$. An LSVDV system is uniformly exponentially stable if there exist $\alpha<1$ and $\beta<\infty$ such that $|x(k)|<\beta \alpha^{k}|x(0)|$. An LSVDV system is stabilizable if there exists $u: \mathbb{R}^{n} \times \Phi \rightarrow \mathbb{R}^{m}$ such that $x(k+1)=A_{\phi(k)} x(k)+B_{\phi(k)} u(x(k), \phi(k))$ is exponentially stable. An LSVDV system is linearly uniformly detectable if there exists a $L: \Phi \rightarrow \mathbb{R}^{n \times p}$ such that the LSVDV system

$$
x(k+1)=\left(A_{\phi(k)}+L_{\phi(k)} C_{\phi(k)}\right) x(k)
$$

is uniformly exponentially stable.

\section{Optimal Controllers for LSVDV Systems are Nonlinear and the Cost is not Quadratic}

Define the terminal cost of the two-step problem as $X(x, \phi, 2,2):=x^{\prime} x$. Then the one-step optimal cost for an LSVDV system is

$$
\begin{aligned}
& X(x, \phi, 1,2)=x^{\prime} C_{\phi}^{\prime} C_{\phi} x \\
& +\min _{u}\left(u^{\prime} D_{\phi}^{\prime} D_{\phi} u+\left(A_{\phi} x+B_{\phi} u\right)^{\prime}\left(A_{\phi} x+B_{\phi} u\right)\right)
\end{aligned}
$$

The worst case two-step optimal cost is

$$
\begin{aligned}
& X(x, \phi, 0,2)=x^{\prime} C_{\phi}^{\prime} C_{\phi} x \\
& +\min _{u} \max _{\rho \in f(\phi)}\left(u^{\prime} D_{\phi}^{\prime} D_{\phi} u+X\left(A_{\phi} x+B_{\phi} u, \rho, 1,2\right)\right)
\end{aligned}
$$

Hence the two-step optimal control problem is a minimax problem where the parameters over which maximization is carried out enters nonlinearly into the cost. Hence, even in this simple two-step problem, the minimizing control is nonlinear in general.

A simple example shows why the worst case cost is not quadratic. Define the LSVDV system with no inputs:

$$
\begin{aligned}
& C=I, A_{a}=\left[\begin{array}{ll}
0 & 0 \\
0 & \alpha
\end{array}\right], A_{b}=\left[\begin{array}{cc}
\alpha & 0 \\
0 & 0
\end{array}\right], \\
& A_{c}=R^{\prime}\left[\begin{array}{ll}
0 & 0 \\
0 & \alpha
\end{array}\right] R, A_{d}=R^{\prime}\left[\begin{array}{ll}
\alpha & 0 \\
0 & 0
\end{array}\right] R,
\end{aligned}
$$

where

$$
R=\left[\begin{array}{ll}
\cos \left(\frac{\pi}{4}\right) & \sin \left(\frac{\pi}{4}\right) \\
-\sin \left(\frac{\pi}{4}\right) & \cos \left(\frac{\pi}{4}\right)
\end{array}\right]
$$

Let

$$
f(a)=f(b)=\{c, d\} \text { and } f(c)=f(d)=\{a, b\} .
$$




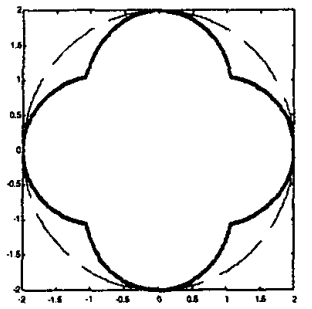

Figure 1: A graphical portrayal of the cost for $\alpha=1$. The above solid line shows a polar plot of $\{(X(x, c, N-2, N) x):\|x\|=1\}$. A polar plot of a quadratic function is an ellipse; hence, $X$ is not quadratic. The dotted line is the least upper quadratic bound on $X$.

Then, with $\phi(0)=c$, the worst case, two-step cost is

$$
\begin{aligned}
& X(x, c, 0,2)=x^{\prime} x \\
& +\max \left(\left|\left[\begin{array}{ll}
0 & 0 \\
0 & \alpha
\end{array}\right] A_{c} x\right|^{2},\left|\left[\begin{array}{cc}
\alpha & 0 \\
0 & 0
\end{array}\right] A_{c} x\right|^{2}\right) \\
= & x^{\prime} x+\max \left(X\left(A_{c} x, a, 1,2\right), X\left(A_{c} x, b, 1,2\right)\right) .
\end{aligned}
$$

Figure (1) shows that this cost is not quadratic. Figure (1) also shows the least quadratic upper bound on $X(x, c, 0,2)$. Note that for $x=\left[\begin{array}{ll}\sqrt{2} & \sqrt{2}\end{array}\right]^{\prime}$, this upper bound is significantly larger than the actual cost. An LMI approach to this problem would use this upper bound as an approximation of the twostep cost. Hence, this cost would be conservative for $x=\left[\begin{array}{ll}\sqrt{2} & \sqrt{2}\end{array}\right]^{\prime}$.

This conservativeness becomes important when testing for stability. From a dynamic programming perspective, the LMI approach approximates the cost with a quadratic function at every step. The conservativeness can accumulate. For the above system given by (2) (4), the LMI approach attempts to find positive semidefinite matrices, $Q_{a}, Q_{b}, Q_{c}$ and $Q_{d}$ such that

$$
\begin{array}{ll}
Q_{a} \geq A_{a}^{\prime} Q_{c} A_{a}+I, & Q_{a} \geq A_{a}^{\prime} Q_{d} A_{a}+I \\
Q_{b} \geq A_{b}^{\prime} Q_{c} A_{b}+I, & Q_{b} \geq A_{b}^{\prime} Q_{d} A_{b}+I \\
Q_{c} \geq A_{c}^{\prime} Q_{a} A_{c}+I, & Q_{c} \geq A_{c}^{\prime} Q_{a} A_{c}+I \\
Q_{d} \geq A_{d}^{\prime} Q_{a} A_{d}+I, & Q_{d} \geq A_{d}^{\prime} Q_{b} A_{d}+I
\end{array}
$$

This is not possible for $\alpha \geq 1$. However, this system is stable for $\alpha<\sqrt{2}$. The method discussed next is not conservative and can be used to show that the above system is stable for $\alpha<\sqrt{2}$.

\section{Analysis for LSVDV Systems}

A nonconservative approach to determine stability of an LSVDV system is to find a positive definite Lyapunov function $X: \mathbb{R}^{n} \times \Phi \rightarrow \mathbb{R}$ such that

$$
X(x, \phi)=\max _{\rho \in f(\phi)} x^{\prime} C_{\phi}^{\prime} C_{\phi} x+X\left(A_{\phi} x, \rho\right) .
$$

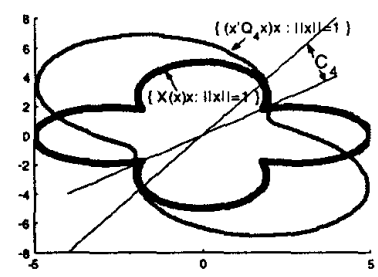

Figure 2: This figure shows $Q_{4}$ a quadratic approximation (thin line) of $X$ (bold line) in the cone $C_{4}$. Note that for $x \notin C_{4}, x^{\prime} Q_{4} x$ is far larger than $X(x)$. Since $\mathcal{Q}(x)=\max _{i} x^{\prime} Q_{i} x \geq x^{\prime} Q_{4} x, \mathcal{Q}$ would not be a very good approximation of $X$.

Similarly, an optimal control can be found by finding a function $X: \mathbb{R}^{n} \times \Phi \rightarrow \mathbb{R}$, such that

$$
\begin{aligned}
& X(x, \phi)= \\
& \min _{u} \max _{\rho \in f(\phi)} x^{\prime} C_{\phi}^{\prime} C_{\phi} x+u^{\prime} D_{\phi}^{\prime} D_{\phi} u+X\left(A_{\phi} x+B_{\phi} u, \rho\right) .
\end{aligned}
$$

There are many potential difficulties with this approach. First, the function $X$ needs to be determined for every $x \in \mathbb{R}^{n}$ and $\phi \in \Phi$. Second, the minimization may be nonconvex and, hence, very difficult to solve. In the LSVDV problem, these difficulties are manageable. However, it must be stressed that this control problem remains difficult; this is essentially a nonlinear control problem.

Next the cost and control will be characterized.

Theorem 1 Let $f$ be a continuous set-valued function, let $A, B, C$ and $D$ be continuous functions and let the $L S V D V$ system be stabilizable, linearly uniformly detectable and let $D_{\phi}^{\prime} D_{\phi}>0$. Then the optimal cost $X: \Phi \times \mathbb{R}^{n} \rightarrow \mathbb{R}$ is uniformly continuous.

Proof: The proof is similar to case where $f$ is single valued which is proved in [5].

The continuity of $X$ is important. It means that the cost, and, hence, the controller, does not need to be found everywhere. Rather, a good approximation can be found by gridding the space $\Phi \times \mathbb{R}^{n}$ and computing $X$ on this grid.

Theorem 2 The optimal control is homogeneous, but, in general, not additive, i.e. $u(\alpha x, \phi)=\alpha u(x, \phi)$, but $u(x+y, \phi) \neq u(x, \phi)+u(y, \phi)$. Similarly, the optimal cost obeys $X(\alpha x, \phi)=\alpha^{2} X(x, \phi)$.

This theorem reduces the dimension by 1 . That is $X$ : $\mathbb{R} \mathbb{P}^{n-1} \times \Phi \rightarrow R$ where $\mathbb{R} \mathbb{P}^{n-1}$ is the $n-1$ dimensional real projective space.

To simplify, an approximate problem will be formulated and its solution will be found. This approximation will converge to the actual solution. The idea is to approximate the cost $X$ by a set of quadratic functions. To 
this end define the function $\mathcal{Q}(x, \phi)=\max _{i} x^{\prime} Q^{i}(\phi) x$, where the $\left\{Q^{i}(\phi): 0<i \leq N\right\}$ 's are such that

$$
X(x, \phi) \approx \mathcal{Q}(x, \phi) \text { for } x \in \mathbb{R} P^{n-1} \text { and } \phi \in \Phi .
$$

We refer to such an approximation as a piece-wise quadratic approximation. This approximation is carried out by partitioning $\mathbb{R}^{n}$ into cones $\left\{\mathcal{C}^{i}\right\}$ and finding a $Q^{i}(\phi)$ such that $X(x, \phi) \approx x^{\prime} Q^{i}(\phi) x$ for $x \in \mathcal{C}^{i}$. However, to make the approximation a good one, $Q^{i}(\phi)$ must be chosen so that it is the least quadratic upper bound of $X$ for all $x \in \mathcal{C}^{i}$. On the other hand, it is desirable that $x^{\prime} Q^{i}(\phi) x \leq x^{\prime} Q^{j}(\phi) x$ for $x \in \mathcal{C}^{j}$. Figure 2 shows a $Q$ where $x^{\prime} Q x \approx X(x)$ for $x \in \mathcal{C}^{4}$, but $x^{\prime} Q x \gg X(x)$ for some $x \notin \mathcal{C}^{4}$. To see how these two requirements can be combined, assume that $\mathcal{C}^{1}$ is the cone centered around $e_{1}:=\left[\begin{array}{llll}1 & 0 & \cdots & 0\end{array}\right]^{\prime}$. In this case,

$\mathcal{C}^{1}:=\left\{\alpha x: \alpha \in \mathbb{R}, x=e_{1}+\varepsilon y: y_{1}=0\right.$ and $\left.|y|=1\right\}$.

Note that the size of the cone is controlled by $\varepsilon$. The matrix $Q^{1}(\phi)$ can be found by solving the following convex minimization problem:

$$
Q^{1}(\phi)=\underset{Q=Q^{\prime} \in \mathbb{R}^{n \times n}}{\arg \min } R Q_{1,1}-\sum_{j>1} \log \left(1-Q_{j, j}\right)
$$$$
\text { subject to : } X(x, \phi) \leq x^{\prime} Q x \text { for all } x \in \mathcal{C}^{1} \text {, }
$$

where $0<R \in \mathbb{R}$. The $R Q_{1,1}$ term forces $Q_{1,1}$ to be small, while the $-\sum_{j>1} \log \left(1-Q_{j, j}\right)$ term avoids problems such as the one shown in Figure 2. Note that the diagonal elements of $Q^{1}(\phi)$ may be negative. That is, the matrix $Q^{1}(\phi)$ may be sign indefinite. By a change of coordinates, this minimization can be repeated for each $\mathcal{C}^{i}$. The resulting piece-wise quadratic approximation is denoted as

$$
\mathcal{Q}_{\varepsilon}(x, \phi):=\max _{i} x^{\prime} Q^{i}(\phi) x .
$$

By making $\varepsilon$ small, this approximation can be made arbitrarily accurate.

Theorem 3 Let $X$ be continuous. Then $\mathcal{Q}_{\varepsilon}(x, \phi)-$ $X(x, \phi) \downarrow 0$ as $\varepsilon \rightarrow 0$ and $R \rightarrow \infty$. In particular, if $\mathcal{C}^{1}$ is defined as in $(5)$ and $Q^{1}(\phi)=\left[\begin{array}{ll}Q_{1,1}^{1}(\phi) & \cdots \\ \vdots & \ddots\end{array}\right]$, then

$$
0 \leq Q_{1,1}^{1}(\phi)-X\left(e_{1}, \phi\right) \leq\left(\frac{1}{R}+\delta(\varepsilon)+\varepsilon^{2}\right)
$$

where

$$
\delta(\varepsilon)=\max _{y \in S^{n-2}}\left|X\left(e_{1}+\varepsilon\left[\begin{array}{l}
0 \\
y
\end{array}\right], \phi\right)-X\left(e_{1}, \phi\right)\right|
$$

Figure 3 shows a 2-D example of this approximation. Note that $\left|Q_{1,1}^{1}-X\left(e_{1}, \phi\right)\right|$ depends on $R$, the weight-

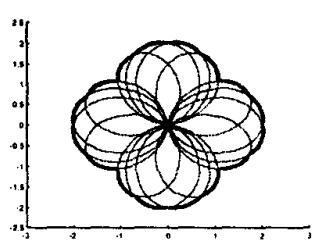

Figure 3: Locally quadratic approximation of a nonquadratic function. This plot shows $\left\{\left(\max _{i} x^{\prime} Q_{i} x\right) x:\|x\|=1\right\}$. Also shown are the plots of $\left\{\left(x^{\prime} Q_{i} x\right) x:\|x\|=1\right\}$ for each $i$.

ing on $Q_{1,1}^{1}$, the continuity of $X$, the function to be approximated, and on $\varepsilon$, the size of the cone. Recall that Theorem 1 states that $X$ is continuous.

The analysis problem can be solved via dynamic programming. To this end, define $X(x, \phi, k, N)$ to be the cost-to-go from step $k$ to step $N$. Hence,

$X(x, \phi, k, N)=x^{\prime} C_{\phi}^{\prime} C_{\phi} x+\max _{\rho \in f(\phi)} X\left(A_{\phi} x, \rho, k+1, N\right)$

It is assumed that the terminal cost is quadratic, e.g. $X(x, \phi, N, N)=x^{\prime} x$. The analysis objective is to find $\mathcal{Q}_{\varepsilon}(x, \phi, k, N)$, a piece-wise quadratic approximation of $X$, such that

$$
X(x, \phi, k, N) \approx \mathcal{Q}_{\varepsilon}(x, \phi, k, N),
$$

where the approximation can be made arbitrarily accurate. Then, by taking $N$ large, the stability can be assessed nonconservatively. Such an approximation is guaranteed by the following theorem.:

Theorem 4 Consider the LSVDV system with $f$ a continuous set-valued map, and $A$ and $C$ continuous. Then, for $N<\infty$, and any $\delta>0$, there exist an $\varepsilon>0$ and an $R<\infty$, such that

$$
\left|X(x, \phi, 0, N)-\mathcal{Q}_{\varepsilon}(x, \phi, 0, N)\right|<\delta,
$$

and, for $\theta \in f(\phi)$ and $\|x\|=1$,

$0 \leq \mathcal{Q}_{\varepsilon}(x, \phi, k-1, N)-\left(x^{\prime} C_{\phi}^{\prime} C_{\phi} x+\mathcal{Q}_{\varepsilon}(A x, \theta, k, N)\right)<\delta$.

\section{Synthesis for LSVDV Systems}

While the analysis problem may be computationally intensive, it is straight forward in the sense that it is a sequence of convex optimization problems. The synthesis problem, however, is not convex. This is the case even when determining the optimal linear controller $F_{i}$ for the piece-wise quadratic approximation problem above, that is, the optimal control for all $x \in \mathcal{C}^{i}$. In fact, computing the control for a specific $x$ is nonconvex. However, the control for a specific $x$ can be found by solving a combination of convex problems. Therefore, a synthesis approach is as follows: 1 . Compute $u\left(x_{j}^{i}, \phi\right)$ 's for a 
linearly independent set $\left\{x_{j}^{i}: j=1, \cdots, N \geq n\right\} \subset \mathcal{C}^{i}$. (This problem will be solved below.) 2. Based on these $u\left(x_{j}^{i}, \phi\right)$, find $F_{\phi}^{i}$ such that $F_{\phi}^{i} x_{j}^{i} \approx u\left(x_{j}^{i}, \phi\right)$. (This can be done using least squares.) 3. Repeat the above for $i=1, \cdots, M$ where the partition of $\mathbb{R}^{n}$ is $\left\{\mathcal{C}^{i} ; i=1, \cdots, M\right\}$. 4. Repeat the above on a grid of $\Phi$.

As stated above, determining $u(x, \phi)$ is nonconvex. To see this suppose that

$$
\mathcal{Q}(x, f(\phi))=\left\{x^{\prime} Q^{1} x, x^{\prime} Q^{2} x\right\}
$$

and

$$
\begin{aligned}
A & =\left[\begin{array}{ll}
-2 & 3 \\
1 & 2
\end{array}\right], B=\left[\begin{array}{l}
1 \\
0
\end{array}\right] \\
C & =\left[\begin{array}{ll}
1 & 0 \\
0 & 1
\end{array}\right], D=1, x=\left[\begin{array}{l}
1 \\
0
\end{array}\right] \\
\text { with } Q^{1} & =\left[\begin{array}{ll}
\frac{1}{2} & 0 \\
0 & 0
\end{array}\right], Q^{2}=\left[\begin{array}{ll}
-2 & -4 \\
-4 & 4
\end{array}\right]
\end{aligned}
$$

Then

$$
\begin{aligned}
& J(x, u, \phi) \\
= & \max \left(x^{\prime} x+u^{\prime} u+\left(A_{\phi} x+B u\right)^{\prime} Q^{1}(A x+B u),\right. \\
& \left.x^{\prime} x+u^{\prime} u+\left(A_{\phi} x+B u\right)^{\prime} Q^{2}(A x+B u)\right) \\
= & \max \left(5-4 u+2 u^{2},-u^{2}+13\right) \\
= & \max \left(J_{1}\left(\left[\begin{array}{ll}
1 & 0
\end{array}\right]^{\prime}, u, \phi\right), J_{2}\left(\left[\begin{array}{ll}
1 & 0
\end{array}\right]^{\prime}, u, \phi\right)\right) .
\end{aligned}
$$

Figure 4 shows plots of $J_{1}$ and $J_{2}$. The worst case cost is the maximum of these two graphs and is not convex. Clearly, the minimum cost is attained at $u=2.5$. However, there is a local minimum at $u=-1$. An algorithm that will always find the global minimum is one that starts at the maximum of $J_{2}$ and performs steepest descent in both directions. In higher dimensions the solution is similar. Except at intersections, the cost hyper-surface has the form $\left(u,\left(u-u^{*}\right)^{\prime}\left(D^{\prime} D+B^{\prime} Q^{i} B\right)\left(u-u^{*}\right)\right)$, where $u^{*}$ is the control given by the standard LQ Riccati equation. This surface has ridges along the lines where (i) $\left(u-u^{*}\right)$ is an eigenvector of $\left(D^{\prime} D+B^{\prime} Q^{i} B\right)$ and (ii) $J_{i}(x, u, \phi) \geq J_{j}(x, u, \phi)$ for all $j$, that is, $J_{i}$ is the active cost. A global minimum can be found by starting the steepest descent along these ridges.

\section{References}

[1] P. Apkarian. Advanced gain-scheduling techniques for uncertain systems. IEEE Transactions on Control Systems Technology, 6(1):21-32, 1998.

[2] P. Apkarian, P. Gahinet, and G. Becker. Selfscheduled $H_{\infty}$ control of linear parameter-varying systems: Design example. Automatica, 31(9):1251-1261, 1995.

[3] J.-P. Aubin and H. Frankowska. Set-Valued Analysis. Birkhauser, 1990.

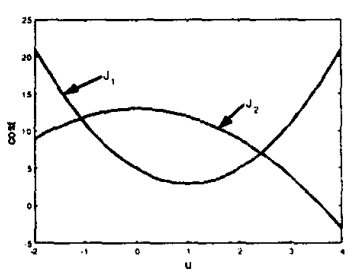

Figure 4: The worst case cost is not convex in the control.

[4] G. Becker and A. Packard. Robust performance of linear parameterically varying systems using parametrically-dependent linear feedback. Systems and Control Letters, 23:205-215, 1995.

[5] S. Bohacek and E. Jonckheere. Linear dynamically varying LQ control of nonlinear systems. Submitted to IEEE Transactions on Automatic Control.

[6] S. Bohacek and E. Jonckheere. Linear dynamically varying (LDV) systems versus jump linear systems. In American Control Conference, pages 4024 4028, San Diego, CA, June 2-4, 1999.

[7] S. Bohacek and E. Jonckheere. Nonlinear tracking over compact sets with linear dynamically varying $H^{\infty}$ control. SIAM Journal of Control and Optimization, (Submitted).

[8] I. Borno and Z. Gajic. Parallel algorithm for solving coupled algebraic Lyapunov equations of discretetime jump linear systems. Computers Mathematics and Applications, 30:1-4, 1995.

[9] S. Boyd, L. E. Ghaoui, E. Feron, and V. Balakrishnan. Linear Matrix Inequalities in System and Control Theory. SIAM, 1994.

[10] O. Costa and M. Fragoso. Discrete-time LQoptimal control problems for infinite Markov parameter system. IEEE Transactions on Automatic Control. 40(12):2076-2088, 1995.

[11] P. Gahinet, P. Apkarian, and M. Chilali. Affine parameter dependent Lyapunov function for real parameter uncertianty. In $C D C .1994$.

[12] Y. Ji, H. Chezeck, X. Feng, and K. Loparo. Stability and control of discrete-time jump linear systems. Control-Theory and Advanced Technology, 7(2):247$270,1991$.

[13] J. S. Shamma and D. Xiong. Set-valued methods for linear parameter varying systems. Automatica, 35:1081-1089, 1999.

[14] F. Wu, X. H. Yang, A. Packard, and G. Becker. Induced $L_{2}$ norm control for LPV systems with bounded parameter variation rates. International Journal of Robust and Nonlinear Control, 6:983-998, 1996. 